\title{
Research Paper: Designing a Computerized Neuro-Cognitive Program for Early Diagnosing Children at Risk for Dyslexia

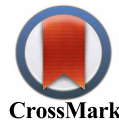

\author{
Mona Delavarian $^{1^{*}}$, Ali Afrooz ${ }^{1}$, Farzad Towhidkhah ${ }^{2}$, Kazem Rasoolzadeh Tabatabaei $^{3}$ \\ 1. Department of Psychology, Faculty of Psychology and Education, University of Tehran, Tehran, Iran. \\ 2. Department of Biomedical Engineering, Amirkabir University of Technology, Tehran, Iran. \\ 3. Department of Psychology, School of Humanities, Tarbiat Modares University, Tehran, Iran
}

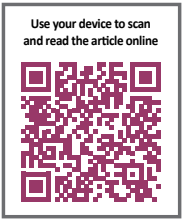

chtation: Delavarian M, Afrooz A, Towhidkhah F, Rasoolzadeh Tabatabaei K. Designing a Computerized Neuro-Cognitive Program for Early Diagnosing Children at Risk for Dyslexia. Iranian Rehabilitation Journal. 2017; 15(2):103-110. https://doi. org/10.18869/NRIP.IRJ.15.2.103

https://doi.org/10.18869/NRIP.IRJ.15.2.103

Article info:

Received: 16 Nov. 2016

Accepted: 25 Feb. 2017

\section{Keywords:}

Neuro-cognitive program, Computer-based task, Working memory, At risk children, Reading disorder (Dyslexia)

\section{A B S T RACT}

Objectives: The aim of this research is to design a neuro-cognitive program, based on dysfunctions and alterations of some neural circuits in dyslexics. The visual and auditory working memories in pre-schoolers were evaluated with this program in order to early screening for dyslexia.

Methods: This study is a longitudinal descriptive research. A total of 259 randomly selected pre-schoolers, with an average age of 6 years, participated in the study and the unskilled data of these students were gathered. These subjects were followed for two years. Thereafter, the remaining subjects were assessed accurately for dyslexia using different reading diagnostic tests. Then, the preschool unskilled data and the diagnosis were applied to automate the designed program. The program was designed in a game format and consisted of separate tasks measuring visual and auditory working memories and rapid naming.

Results: From the calculation of content validity rate as the first step of content validity process, 3 out of 5 components, including visual and auditory working memories, and rapid naming were selected. The process and the results of content validity showed that the designed program has an appropriate and acceptable level of content validity. The amount of reliability of the program for visual and auditory working memories, and rapid naming were obtained as $0.89,0.92$ and 0.88 , respectively. Moreover, the discriminative validity for auditory and visual working memories and rapid naming, between children with dyslexia, Attention Deficit Hyperactivity Disorder (ADHD) (a disorder having high similarity with dyslexia) and normal ones were obtained as $0.87,0.67$ and 0.8 , respectively. It is worthy to say that the prediction accuracy of this neuro-cognitive program for dyslexia achieved $90.65 \%$.

Discussion: This program has an acceptable validity and reliability. It could be useful as an accurate assessment tool in predicting dyslexia before the occurrence of psychological scars and can be used as a quick screening tool for children at risk for dyslexia. 


\section{Introduction}

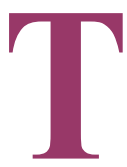

he early and accurate diagnosis of "specific learning disorders" is one of the major concerns in the field of children psychology. Learning difficulties often lead to lack of motivation, low self-esteem, persistent feeling of failure and poor relationships with peers. Furthermore, these disorders increase the risk of Attention Deficit Hyperactivity Disorder (ADHD), depression, communication and conduct disorders [1]. Prevalence of specific learning disorders in different languages and cultures is 5 to $15 \%$ among school-aged children [2].

In recent years, researchers have shown that the majority of students with specific learning disabilities have problems in reading as one of the main learning pre-requisites [3]. Dyslexia or "reading disorder" affects $15 \%$ of children worldwide [4]. Dyslexia is related to a deficiency in precise and explicit decoding and weak spelling of words [2]. American national institute of health has defined dyslexia mainly as word recognition problem, particularly in the areas of spelling, perception and reading comprehension.

With early diagnosis and intervention, dyslexia could be conquered; otherwise, it would become a life-long problem [5]. In addition, reading proficiently is a prerequisite to fit in the modern civilized societies, and therefore, a dyslexic person may encounter social, educational, emotional as well as psychological problems. For instance, dyslexic children's failures in school might influence their learning motivation, educational opportunities and quality of life [6] that would lead to lower school achievements as compared to other classmates, failure, dropout [7], frustration [8], and losing opportunities [9]. The sense of frustration may increase gradually and eventually lead to poor self-esteem causing inappropriate behavior in school. It is shown that the dropout rate is higher in dyslexics as compared to the other learning disabilities [10].

All of the above mentioned problems are preventable with an early diagnosis and intervention. Unfortunately, no unique symptom assists in the early and accurate diagnosis of dyslexia. The comparison of children's expected and actual school achievement by standardized tests is the common diagnostic approach that most experts follow [11]. Consequently, dyslexia is commonly discovered during elementary school, where the reading ability is essential for success in other subjects [12]. Moreover, the diagnosis with comprehensive assessment method needs trained professionals [10] who are not accessible for many children, and even if present, no consensus exists among them in many instances [13].
Therefore, in this research, we have designed a computer based screening program that can be used as a prediction method in schools in the absence of trained professionals. We have focused on neuro-cognitive impairments of dyslexic individuals, instead of reading ability, since it is believed that dyslexia is the result of some neural circuits dysfunctions and alterations [14], such as Fronto-striatal circuits [15], left arcuate fasciculus [16], and corpus callosum [14]. Visual and auditory working memories are among the most highlighted affected cognitive skills due to these alterations [17]. A significant reduction in working memory function and capacity has also been ascribed to dyslexia [18-20].

We have designed a screening tool for dyslexia based on feeding a knowledge base of impaired executive functions in dyslexia to a neuro-cognitive computer program with a game frontend. This includes visual and auditory working memories as two important components of assessment. Furthermore, the task for rapid naming measurement was included in order to customize the program for dyslexia [21] and separate it from other learning disabilities.

\section{Methods}

\section{Research design}

This is a longitudinal descriptive research in terms of its method of data collection and Research and Development (R\&D) study, in terms of its goal. The designed program is in a game format to motivate subjects. C language was used for this purpose because of its overall performance. The design is focused on neuro-cognitive impairments due to mentioned neural circuits' dysfunctions and alterations in dyslexia; therefore, this program is able to screen or predict dyslexia regardless of reading skills; separate tasks were designed to evaluate visual and auditory working memory.

\section{Auditory working memory task}

After hearing the name of a set of objects, subjects were observing a set of related pictures on a monitor. Children were then asked to point to the objects in the monitor in the same order, which they had heard earlier. The score was calculated by success in remembering pictures in the correct order at any stage.

\section{Visual working memory}

Test subject should remember the places and directions of a set of objects that appeared for a short time on the monitor, and subsequently test subjects were asked to 
point the place of one of them. In both of these tasks, the number of items that were heard and seen initially started with two objects and increased further according to the success in each stage. The process was continued until two sequential failures happened. Children's cognitive performance was calculated based on the number of correct clicks and reaction times, which were saved in a spreadsheet.

\section{Rapid naming task}

A full page of different well-known fruits (such as apple, grape, cherry, orange and banana) was displayed for each subject. Before starting this task, the pictures were identified for each individual to ensure that they are on the same page in this respect. The duration of this task was 30 seconds and students were asked to name the fruits at maximum speed, as soon as the test started. There were 20 fruits in each line and at the end of the task, the number of correct-mentioned fruits was recorded automatically. Finally, the speed of subjects in naming the pictures was compared.

\section{Validity and reliability evaluations}

The validity of the designed neuro-cognitive program was investigated with the calculation of "content validity" and "discriminative validity", which are defined as follows.

\section{Content validity}

For this purpose first a table of each section's desired components was provided in the form of a questionnaire. Then the program was run part by part for four specialists with at least fifteen years of experience in the field of diagnosis of learning disabilities. The experts were asked to fill a questionnaire in which the compliance of that section of the program with the desired components was ranked from 1 (no compliance) to 10 (high compliance). Finally, the correlation between the scores given by each expert was measured by multivariate correlation coefficient, SPSS (IBM Corp. Released 2013. IBM SPSS Statistics for Windows, Version 22.0. Armonk, NY: IBM Corp) was used for all statistical calculations.

\section{Discriminative validity}

The neuro-cognitive program's ability in distinguishing disorders with similar symptoms with dyslexia such as ADHD was calculated. For this purpose, 15 children with a definitive diagnosis of dyslexia, 13 with ADHD and 15 normal readers were tested by the program, and then the results were compared. The score differences in working memory task were evaluated with Chi-square homogeneity test, and in rapid naming task, with one way ANOVA. SPSS program was used for this purpose.

\section{Program reliability}

The reliability estimation was done with "test re-test method." For this purpose, 30 normal subjects were tested twice by the program in a month interval, with the maximum similarity in implementation. The correlation between the scores in these two steps was measured and Chi-square test (for working memories) and t-test (for rapid naming) were applied to investigate the significance of the correlation coefficient of concordance.

\section{Subjects}

For the present study, pre-schoolers, before learning reading skill, from both sexes were selected from nineteenth districts of Tehran province. Subjects were selected based on cluster random sampling method; firstly, some regions were selected randomly from the list of those nineteenth provinces and then some schools were selected randomly in selected regions, then all the preschoolers in those schools were included in the study. It is worth to mention that all of these subjects were assessed in terms of Intellectual Ability (IQ), in authentic special assessment centers. According to specialists opinion, those subjects falling under the normal range of IQ, less than 80 , were excluded from the study.

A meeting was organized in each of the selected schools to explain the study objectives to parents. At least one of the guardians should be present in that meeting. Moreover, detailed information about demographic and developmental stages of children who attended the meeting was gathered. At the end of the meeting, the designed computer-based neuro-cognitive program was introduced to the guardians and the consent about willingness to participate in the study was granted. Then all the subjects were tested by the designed computerized neuro-cognitive program.

The task performance data and scores were stored in spreadsheets for two years, until a definite diagnosis, i.e., whether dyslexic or normal reader was made for each child. The academic situation of the children was followed every six months during those two years, through their teachers and school staffs to investigate their academic achievements. After two years, at the end of the second grade of elementary school, subjects were evalu- 
ated, in terms of accuracy, speed and comprehension of reading by means of different reading diagnostic tests [3].

\section{Prediction accuracy}

At the end of the second grade of elementary school, each subject received one code, which indicated their diagnosis or output ( 0 for normal readers and 1 for dyslexics). According to the definite diagnosis of each child as the output, the initial or unskilled data, used to automate or train the neuro-cognitive program. The data that belonged to preschools were saved for two years and were free from the influence of interventions and school training. For this purpose, the unskilled data of each pre-schooler and the output of that subject, which was observed at the end of the second grade of elementary school, were entered and identified to the program.

After training phase, in order to estimate the prediction accuracy or test the program, half of the preschoolers' data or unskilled data (107 of them) were randomly selected for 100 times. These unskilled data was entered into the program and the output of the automated computerized neuro-cognitive program, 0 or 1 , was compared to the real diagnosis, which appeared in the second grade of elementary school. The average of the correct number of predictions was considered as the prediction accuracy. The training and testing phases were implemented with artificial neural networks methods from artificial intelligence algorithms through MATLAB software.

\section{Results}

Out of 256 pre-schoolers that initially entered the study, 237 remained in the study after two years of follow-up, and of the 237, 167 were normal readers, 47 dyslexics, and 23 had another disorder such as ADHD, or Oppositional Defiant Disorder (ODD), and therefore, their data were excluded.

In order to evaluate content validity, multivariate correlation was calculated between the compliance score of each section with the desired component, which was given by four experts. Strong correlation existed between these experts' opinions on the compliance of that section. The designed neuro-cognitive program had sig- nificant content validity (Table 1 ). The rate of correlation obtained was $0.68(\mathrm{CR}=0.68)$, which was statistically significant $(\mathrm{P}=0.015)$.

For assessing the discriminative validity, the neurocognitive program was performed by subjects with a definite diagnosis of dyslexia, ADHD and normal readers. The program compared the visual and auditory working memories and rapid naming of these groups. Chi-square homogeneity test was applied in order to investigate the ability of the program in discrimination of these three groups and it showed that the tasks related to auditory working memory have more ability in distinguishing dyslexia from other similar groups; the amount of $\chi^{2}$ obs for auditory working memory was 46.19 in comparison with visual working memory, which was 19.584 . The amounts of $\chi^{2}$ were significant for both auditory and visual working memories; P-values were 0.009 and 0.033 , respectively (Table 2).

In order to investigate the ability of the program in differentiating these groups, according to rapid naming, one-way ANOVA was applied. This task had the strong ability in distinguishing these groups $(\mathrm{F}=81.60)$ and the result was significant $(\mathrm{P}=0.001)$ (Table 3 ).

Test, re-test with one-month intervals were used to investigate the reliability of the designed neuro-cognitive program and the correlation was measured between the scores of these two stages. The correlations obtained was 0.78 and 0.68 for auditory and visual working memories, respectively and 0.88 for rapid naming. The significance of the correlations was investigated by the correlation coefficient of concordance and they were found to be significant in all of these components $(\mathrm{P}=0.001)$ (Table 4).

At the end of the second grade of elementary school, each subject received one code which indicated their diagnosis or output ( 0 for normal readers and 1 for dyslexics). According to the definite diagnosis of each child as the output, the initial or unskilled data was used to automate or train the neuro-cognitive program. Unskilled data belonged to preschools, were saved for two years, and were free from the influence of interventions and school training. For this purpose, the unskilled data of each pre-schooler and the output of that subject, which

Table 1. Multivariate correlation between the experts

\begin{tabular}{ccccc}
\hline $\mathbf{R}$ & R Square & Adjusted R Square & Std. Error of the Estimate & F \\
\hline 0.68 & 0.45 & 0.35 & 0.79 & 4.651 \\
\hline
\end{tabular}


Table 2. Chi-square homogeneity results in discrimination of groups of dyslexia, ADHD and normal subjects

\begin{tabular}{cccc} 
& $\boldsymbol{X}^{2 \text { obs }}$ & df & P \\
\hline Auditory working memory & 46.19 & 26 & 0.009 \\
Visual working memory & 19.584 & 10 & 0.033 \\
\hline
\end{tabular}

Table 3. The result of one way ANOVA in discrimination of groups of dyslexia, ADHD and normal subjects with rapid naming task

\begin{tabular}{cccccc}
\hline & Sum of Squares & df & Mean Square & F & P \\
\hline Between groups & 937.824 & 2 & 468.912 & 81.606 & 0.001 \\
\hline Within groups & 155.143 & 27 & 5.746 & & \\
\hline Total & 1092.967 & 29 & & \\
\hline
\end{tabular}

Table 4. The correlation between the scores of test and re-test

\begin{tabular}{ccc}
\hline & Value of Correlation & Approx. P \\
\hline Auditory working memory & 0.78 & 0.001 \\
\hline Visual working memory & 0.68 & 0.001 \\
Rapid naming & 0.88 & 0.001 \\
\hline
\end{tabular}

appeared at the end of the second grade of elementary school, were entered and identified to the program. After training phase, in order to estimate the prediction accuracy or test the program, half of the preschoolers' data or unskilled data (107 of them) were randomly selected for 100 times. These unskilled data entered into the program and the output of the automated computerized neurocognitive program, 0 or 1, was compared to the real diagnosis, which appeared in the second grade of elementary school. The average of the correct number of predictions was considered as the prediction accuracy.

In order to automate the designed neuro-cognitive program after estimating the validity and reliability of the preschool unskilled data and the definite diagnosis of the second grade of elementary school were considered. In order to calculate the accuracy of the prediction of the automated designed neuro-cognitive program, half of the preschool data and the related diagnostic code $(0$ for normal readers and 1 for dyslexics) were selected. In this way, the unskilled data of 107 subjects, 30 dyslexics and 77 normal readers, which belonged to preschoolers and were saved until the definite diagnosis was made, were entered into the designed program and the output of the automated program was compared to the real diagnosis. This entering and comparing process was repeated 100 times and the proportion of correct prediction, which was considered as the prediction accuracy, reached to $90.65 \%$ with an average of 10 errors. An average of 3 errors in predicting children at risk for dyslexia decreases the prediction sensitivity of the neuro-cognitive program to $91.08 \%$.

\section{Discussion}

Diagnostic tests and psychological assessment tools are designed to reveal disorders, disabilities and specific behaviors. Early screening and diagnosis lead to effective and in time intervention. Each of the diagnostic and screening tools has their own shortcomings. For instance, most of them need the reading [21-24] and they lead to experience many defeats during the years to reveal [24], lack of willingness to accomplish the test because a lot of time is required to complete it, is another disadvantage[25, 26]. Most of the time, the test and the tasks are not attractive because they are just computer-based of the paper-pencil test [27] and therefore cannot motivate subjects to use their maximum potential ability and moreover, although some of the tasks are designed in game format but their validity and reliability are not apparent $[1,28]$.

The aim of this study was to design a computerized neuro-cognitive program for the prediction of dyslexia in children at risk. To achieve the objective, the visual and auditory working memories were measured. According 
to various scientific studies and research, these two important cognitive components are among the most important affected cognitive components in dyslexia [14, 20]. Furthermore, the information processing theory is considered as one of the most authentic theories in this field [29]. Moreover, rapid naming task, was included to customize the program for dyslexia, which separates it from the other neuro-developmental disorders, especially from other learning disabilities [21].

To obtain more accuracy, the maximum potential of visual and auditory working memories and rapid naming were needed and since the subjects were pre-schoolers the program was designed in game format. This is one of the most advantages of this program in comparison with the other screening and predictive tests and tools. Previously, several tests and tools have been introduced to screen and assess dyslexia. Some of them are either pencil-paper tests or computer-based [30].

In these test and screening programs, some cognitive abilities of children with neuro-developmental disorders such as dyslexia are measured and estimated, in addition, some tasks are included for assessing reading besides cognitive abilities $[21,24]$. The target samples are elementary students, and in most of these studies, the subjects are 8 to 11 (or more) years old [22]. These tests are designed for screening dyslexia, with a combination of paper-pencil and computer based program. In these studies, the process of investigation takes a long time $[25,26]$, and it is apparent that completing these test lead to fatigue in children and if they perform for more than one session, these tests will be prone to some errors.

Another advantage of the current designed neurocognitive program is that in previous studies, the tests needed reading ability to fulfill, so the subjects were experiencing many defeats during years to reveal [22]. However, our program assesses some of the important cognitive abilities required for reading ability, besides its suitable format and accuracy (the value of 46.19 and 19.58 for auditory and visual working memories), which were both significant in distinguishing and the amount of 0.55 in differentiation in according to rapid naming task. Stanford-Binet Intelligence scale is another instance which was applied in learning disabilities prediction [31], besides estimating IQ. The previously mentioned hints are consistent in this example too.

\section{Conclusion}

A neuro-cognitive program considering significant differences in working memory and rapid naming tasks
$[16,21,32]$ was designed in order to screen or somehow predict subjects at risk for dyslexia and the program was further investigated for validity and reliability. The results showed that the program has high content validity, according to the experts' opinion, and discriminative validity in distinguishing ADHD, Dyslexia and normal readers. Considering the tasks for separate assessment of visual and auditory working memories is one of the most important advantages of this program, which is not considered in most of the previous studies and other known common diagnostic and screening tools and programs. Furthermore, when it comes to children, the time required for accomplishing and completing the predictive or screening program plays a pivotal role.

A good program is better to be in a game format, so that it will be attractive and encourage active participation, cooperation and tolerance; therefore, the subjects would accomplish the task with the greatest potential abilities. Another important element that was considered in this study, according to the importance of reading ability and many social, educational and psychological side effects of defeats in this field, was screening or somehow prediction of children at risk for dyslexia as soon as possible even before elementary school. In this way, the high-risk subjects are able to be identified, by which more accurate assessments and the early intervention will be done, so that the mentioned side effects will be prevented. Finally, this designed computer-based neuro-cognitive program is recommended to be applied in kindergartens and preschools as a valid and reliable screening or predictive program in order to early diagnosis and interventions of dyslexia and for preventing many side effects.

\section{Acknowledgements}

This research was extracted from Delavarian $\mathrm{PhD}$ thesis (2016) in the Department of Psychology, Faculty of Psychology and Education, University of Tehran, Tehran, Iran.

\section{Conflict of Interest}

All authors certify that this manuscript has neither been published in whole nor in part nor being considered for publication elsewhere. The authors have no conflicts of interest to declare.

\section{References}

[1] Carnine L, Carnine D. The interaction of reading skills and science content knowledge when teaching struggling second- 
ary students. Reading \& Writing Quarterly. 2004; 20(2):20318. doi: $10.1080 / 10573560490264134$

[2] American Psychological Association. Diagnostic and statistical manual of mental disorders. New York: American Psychological Association; 2000.

[3] Awolola SA. Effect of brain-based learning strategy on students' achievement in senior secondary school mathematics in Oyo State, Nigeria. Cypriot Journal of Educational Sciences. $2011 ; 2: 91-106$

[4] Jeyasekaran J. Effectiveness of visual auditory kinesthetic tactile technique on reading level among children with dyslexia at Helikx Open School and Learning Centre, Salem. International Journal of Medical Science and Public Health. 2015; 4(3):315. doi: 10.5455/ijmsph.2015.0511201467

[5] Castro F, Vellido A, Nebot À, Mugica F. Applying data mining techniques to e-learning problems. Studies in Computational Intelligence. 2007; 183-221. doi: 10.1007/978-3-54071974-8_8

[6] Daly EJ. Interventions for reading problems: Designing and evaluating effective strategies. New York: Guilford Publications; 2015.

[7] Biancarosa G, Snow C. Reading next: A vision for action and research in middle and high school literacy. New York: Carnegie Corporation; 2004.

[8] Denton CA, Vaughn S. Reading and writing intervention for older students with disabilities: Possibilities and challenges. Learning Disabilities Research \& Practice. 2008; 23(2):61-2. doi: $10.1111 / \mathrm{j} .1540-5826.2008 .00263 . x$

[9] Torgesen JK. Avoiding the devastating downward spiral The evidence that early intervention prevents reading failure. American Educator. 2004; 28(3):6-19.

[10] Chiappedi M, Zoppello M, Rossi R, Scarabello EM, Piazza F. Specific learning disabilities and psychopathological aspects: The importance of early diagnosis (Italian)]. Minerva Pediatrica. 2007; 59(3):281-7. PMID: 17519874

[11] Lyon GR, Fletcher JM, Shaywitz SE. Rethinking learning disabilities. In: Finn CE, Rotherham AJ, Hokanson CR, editors. Rethinking Special Education for a New Century; 2001.

[12] Julie MD, Balakrishnan K. Significance of Classification Techniques in Prediction of Learning Disabilities. International Journal of Artificial Intelligence \& Applications. 2010; 1(4):111-20. doi: 10.5121/ijaia.2010.1409

[13] Snowling MJ. Early identification and interventions for dyslexia: A contemporary view. Journal of Research in Special Educational Needs. 2012; 13(1):7-14. doi: 10.1111/j.14713802.2012.01262.x

[14] Kershner JR. A mini-review: Toward a comprehensive theory of dyslexia. Journal of Neurology and Neuroscience. 2015; 6(1). doi: 10.21767/2171-6625.s10013

[15] Kudo MF, Lussier CM, Swanson HL. Reading disabilities in children: A selective meta-analysis of the cognitive literature. Research in Developmental Disabilities. 2015; 40:51-62. doi: 10.1016/j.ridd.2015.01.002

[16] Van Dyke JA, Johns CL, Kukona A. Low working memory capacity is only spuriously related to poor reading compre- hension. Cognition. 2014; 131(3):373-403. doi: 10.1016/j.cognition.2014.01.007

[17] Brandenburg J, Klesczewski J, Fischbach A, Schuchardt K, Büttner G, Hasselhorn M. Working memory in children with learning disabilities in reading versus spelling. Journal of Learning Disabilities. 2014; 48(6):622-34. doi: $10.1177 / 0022219414521665$

[18] Roid GH, Tipish A, Pamplin Z, Master FJ. A review of Stanford-Binet intelligence scales, $5^{\text {th }} \mathrm{Ed}$ for use with learning disabilities children. The Journal of Social Psychology. 2011; 36(29):296-302.

[19] Roid GH, Barram RA. Essentials of Stanford-Binet intelligence scales (SB5) assessment. New Jersey: John Wiley \& Sons; 2004.

[20] Vandermosten M, Boets B, Poelmans H, Sunaert S, Wouters J, Ghesquiere P. A tractography study in dyslexia: Neuroanatomic correlates of orthographic, phonological and speech processing. Brain. 2012; 135(3):935-48. doi: 10.1093/brain/ awr363.

[21] Andrade OVCA, Andrade PE, Capellini SA. Collective screening tools for early identification of dyslexia. Frontiers in Psychology. 2015; 5. doi: 10.3389/fpsyg.2014.01581

[22] Vidyasagar N, Bhogle S. ART: A cognitive screening tool for reading and arithmetic difficulties. International Journal of Indian Psychology. 2015; 2(4):7-19.

[23] Georgiou GK, Papadopoulos TC, Zarouna E, Parrila R. Are auditory and visual processing deficits related to developmental dyslexia? Dyslexia. 2012; 18(2):110-29. doi: 10.1002/ dys.1439

[24] Mat NSFN, Shamsuddin SWA, Husain R. A Conceptual framework for designing a computer-based dyslexia screening test. Paper presented at: The $3^{\text {rd }}$ International Conference on Informatics \& Applications (ICIA2014). 2014 October 15; Kuala Terengganu, Malaysia.

[25] Toki, EI, Zakopoulou V, Pange J. Preschoolers' learning disabilities assessment: New perspectives in computerized clinical tools. Sino-US English Teaching. 2014; 11(6):401-10.

[26] Puolakanaho A. Early prediction of reading: Phonological Awareness and related language and cognitive skills in children with a familial risk for dyslexia [PhD dissertation]. Jyväskylä: University of Jyväskylä; 2007.

[27] Wallen NE, Fraenkel JR. Educational research: A guide to the process. Philadelphia: Psychology Press; 2001.

[28] Facoetti, A. Multiplatform games for dyslexia identification in preschoolers. Paper presented at: The Consumer Communications and Networking Conference (CCNC); 2014 IEEE $11^{\text {th }}$; Las Vegas, USA.

[29] Vasic N, Lohr C, Steinbrink C, Martin C, Wolf RC. Neural correlates of working memory performance in adolescents and young adults with dyslexia. Neuropsychologia. 2008; 46(2):640-8. doi: 10.1016/j.neuropsychologia.2007.09.002

[30] Ortiz SO, Flanagan DP, Alfonso VC. Essentials of crossbattery assessment. Vol. 84. Philadelphia: John Wiley \& Sons; 2013.

[31] De Beer J, Engels J, Heerkens Y, van der Klink J. Factors influencing work participation of adults with developmental 
dyslexia: a systematic review. BMC Public Health. 2014; 14:77. doi: 10.1186/1471-2458-14-77

[32] Hachmann WM, Bogaerts L, Szmalec A, Woumans E, Duyck W, Job R. Short-term memory for order but not for item information is impaired in developmental dyslexia. Annals of Dyslexia. 2014; 64(2):121-36. doi: 10.1007/s11881-013-0089-5 